\title{
SYMPOSIUM
}

\section{Gonorrhoea and chlamydia core groups and sexual networks in Manitoba}

\section{A M Jolly, J L Wylie}

Sex Transm Infect 2002;78(Suppl I):i145-i151

This paper summarises the results of the $R_{0}$ equation in sexually transmitted infection (STI) repeaters in Manitoba, Canada, in the early 1990s, with both concurrent and more recent descriptions of sexual networks in the same population. The research presented provides empirical network and sex partner data to refine definitions of sexual networks and core groups in phase IV epidemics. New challenges for both practice and research are also discussed.

See end of article for authors' affiliations

...................

Correspondence to: Dr Ann M Jolly, Bureau of HIV/AIDS, STD and TB,

Room 702A, Jeanne Mance Building, PL1907A4, Tunney's Pasture, Ottawa, Ontario Canada K1A OL2: ann_jolly@hc-sc.gc.ca

Accepted 6 December 2001 $\mathrm{n}$ an effort to reduce the burden and cost of illness of sexually transmitted infections, legislation, policy guidelines, and programmes have been implemented in the developed world. Syphilis and gonorrhoea have been notifiable diseases in many jurisdictions for decades. ${ }^{1}$ Chlamydia was reported in most provinces in Canada by 1991, ${ }^{2}$ and in the USA 46 states reported chlamydial infections to the Centers for Disease Control (CDC) by 1992. ${ }^{3}$ Guidelines on the diagnosis, treatment, and management of gonorrhoea and chlamydia have been published regularly in the USA and Canada. Screening programmes to detect asymptomatic patients with disease have also been implemented. ${ }^{4}$ However, screening and other population based prevention programmes which may be effective in the early stages of disease control become less effective and cost per case rises as the prevalence of the disease decreases. $^{56}$ Recently, the decline in rates of chlamydia in Manitoba, as in other jurisdictions, ${ }^{6-9}$ slowed from 1993 to $1995,{ }^{10}$ and positivity rates for chlamydia laboratory tests in women levelled off to 3.6\% between 1992 and 1994. The incidence of syphilis has been below $1 / 100000$ since $1987 .{ }^{10}$ If gonorrhoea is to be eliminated and if chlamydia incidence is to be further reduced, refinements in control programmes are warranted, and research should be concentrated on understanding why and how sexually transmitted infections (STI) are currently spread in low incidence areas.

Core group theory has been proposed as a useful framework to help focus research and prevention efforts on mechanisms of STI transmission. It is hypothesised that a small subset (core group) of the population with large numbers of partners and relatively long periods of infectiousness contribute disproportionately to the spread of STIs. ${ }^{11}$ The first description of the "core group" occurred in 1978, stimulated by the discovery that when screening for gonorrhoea was introduced in the USA, the detection of an additional 10\% of gonococcal infections in women resulted in a $20 \%$ decrease of infections in the years immediately following. ${ }^{12}$

The basic formula for describing the success of a disease in a population (that is, whether it propagates or dies out), is:

$$
R_{0}=\beta c D
$$

$\mathrm{R}_{0}$ is the reproductive number of infection and represents the "average number of secondary infections produced when one infected individual is introduced into a host population where everyone is susceptible." ${ }^{13}$ If $\mathrm{R}_{0}$ is greater than 1 , such as within the core group, the disease will spread in a population; if it is consistently less than 1 , the disease will eventually cease to exist in the population. ${ }^{14}$ The term $\beta$ is the probability of transmission from one infected individual to a susceptible partner; $D$ denotes the duration of infectiousness, and $c$ is the measurement of random sexual contact between infectious individuals and susceptible individuals given by:

$$
c=m+\frac{\sigma^{2}}{m}
$$

where $m$ is the mean number of new partners exposed per unit of time, and $\sigma^{2}$ is the variance of the population mean. Obviously of more concern are those who are "super spreaders", ${ }^{15}$ as they are responsible, directly and indirectly, for the progression of disease through a population. If they have sex only with one another, there would be no disease outside that group. If they mix only with people who do not have large numbers of partners, the infection would die out, because their partners do not have enough partners during the infectious period to transmit the infection further.

Wasserheit and Aral have used the equation and core group concept in defining stages of STI epidemics, from initial introduction and rise of a pathogen in a susceptible population $\left(\mathrm{R}_{0}>1\right)$, the growth of the epidemic to its highest level $\left(R_{0}=1\right)$, and, as diagnostics and treatment methods are developed and applied, the decline of the epidemic $\left(\mathrm{R}_{0}<1\right)$ until a plateau is reached where lower level endemicity is maintained $\left(\mathrm{R}_{0}=1\right){ }^{6}$ In addition, they have proposed that two structurally different types of sexual network with varying capacities for pathogen spread constitute core groups, causing the number of cases to rise (spread networks $\left.\left(R_{0}>1\right)\right)$ or level off (maintenance networks $\left.\left(\mathrm{R}_{0}=1\right)\right)$. Other networks, in which the reproductive number is less 
than 1, do not support the transmission of the pathogen and it dies out (dead end networks). ${ }^{16}$

Accordingly, in this paper we summarise the results of the $\mathrm{R}_{0}$ equation in STI repeaters in Manitoba, Canada, in the early 1990s, with both concurrent and more recent descriptions of sexual networks in the same population. In addition to the evidence of a plateau in STI incidence reported above, Elliott $e t$ al have presented other analyses ${ }^{17}$ which establish that gonorrhoea and chlamydia in Manitoba have been in the low incidence endemic phase (phase IV), as defined by Wasserheit and Aral, since 1990. The research presented below provides empirical network and sex partner data to refine definitions of sexual networks and core groups in phase IV epidemics. New challenges for both practice and research are also discussed. Syphilis has been omitted from this discussion, as there have been very few cases in Manitoba recently (see above.)

\section{METHODS}

The study on core group membership and those on sexual networks rely heavily on the Cadham Provincial Laboratory (CPL) information system and on notifiable disease data collected in a registry under the Public Health Act and regulations of the Province of Manitoba. The data sources and the management of people with STIs and their partners have been described elsewhere. ${ }^{18}{ }^{19}$ Briefly, sexually transmitted diseases in Manitoba are screened, diagnosed, and treated by physicians and nurses practising in medical clinics and hospitals. There are no designated sexually transmitted disease clinics in the province. Diagnosticians and laboratories are responsible for reporting both chlamydial and gonococcal infections to the Manitoba Communicable Disease Control (MCDC) of Manitoba Health. Notification of potentially exposed sex partners is completed by the health care provider or by the patients themselves. In the latter case, the health care provider may note the names to ensure that the partners presented for testing and treatment-that is, "contracting". If this fails to occur, the care provider in these instances personally locates and notifies the partners. In the case of health care provider referral, and in the latter "contracting" method, named sex partners are recorded in the contact registry if they had sex with the case one incubation period before symptoms began, and before the case had been treated. In the absence of specific information on exposure and treatment (by far the majority of instances), sex partners were recorded as contacts if they had sex with a case in the last three months. ${ }^{20}$

Computed laboratory information was used, in addition to the notifiable sexually transmitted disease (STD) registry. Ninety five per cent of the screening and diagnostic tests for genital chlamydial infections were carried out at Cadham Provincial Laboratory. Screening tests were recommended for all women with multiple sex partners, for those with a recent history of, or exposure to, an STI, and for women undergoing therapeutic abortions or intrauterine device insertion. Screening of sexually active women under the age of 25 was added in 1989 to the Canadian chlamydia screening guidelines, but was not recommended for women presenting for annual or prenatal examinations unless they met the above criteria. Computed laboratory records on each specimen contained information on the specimens, dates, types of tests completed, physician information, and patient information.

\section{STI CORE GROUPS IN MANITOBA}

One of the objectives in our initial research was to calculate the reproductive numbers $\left(\mathrm{R}_{0}\right)$ of core and non-core groups to examine the persistence of gonorrhoea and chlamydia in different segments of the Manitoba population. If successful, the results could guide public health professionals in refining interventions for core groups-a more efficient prevention strategy than targeting whole populations. Clients testing positive for chlamydia, gonorrhoea, and both infections more than once during the study period 1990-92 (repeaters) were designated core group members. ${ }^{121-24}$ Those with only one episode of infection were considered to be non-core-group members. In order to gain a perspective on the context in which disease transmission was taking place, we also wished to establish the feasibility of constructing networks from contact tracing data.

Sex partner data from the notifiable STD registry, and duration and transmission probabilities from published reports, were used to calculate $\mathrm{R}_{0}$ for the proposed core groups, as neither was available from our data. The probability of $0.10(\beta)$ for transmission of chlamydia during one episode of sexual intercourse ${ }^{25}$ was taken from that estimated by Brunham and Plummer, ${ }^{14}$ and adjusted by Stigum et al. ${ }^{26}$

The duration time of 10 months for chlamydia was obtained from a study in which infectious days of the women in the study could be calculated. ${ }^{27}$ The transmission probability for gonorrhoea of 0.43 is an average of the probabilities of transmission from a single episode of intercourse from women to men $(0.25)$ and from men to women $(0.60)$, and was taken from studies of exposure of naval personnel to infected sex workers $^{28-30}$ and from early reports of mathematical models. ${ }^{31}$

The duration time of 55 days for gonorrhoea was taken from early estimates of duration of gonorrhoea in the presence of a control programme. ${ }^{12}$ In the absence of transmission probabilities of co-infection between couples, we have used an older estimate of gonorrhoea transmission of $0.5,{ }^{30}$ a higher probability than that used for gonococcal infection above, but biologically plausible, as the release of bacteria from tissue disrupted by two infections instead of one may result in a higher dose and more effective transmission. The duration time for gonorrhoea was used in calculating $\mathrm{R}_{0}$ for the co-infected repeaters, as co-infected people were more likely to report symptoms, for which they were more likely to seek care, thus limiting the period of infectiousness to a shorter period than chlamydial infection alone.

$\mathrm{R}_{0}$ values for repeaters in all three disease categories approached or exceeded unity, while those for non-repeaters

Table 1 Mean rates of partner change $(m-1)$, variance $\left(\sigma^{2}\right)$ of the number partners, and average $R_{0}$ for female and male subjects in the study populations, showing higher partner change rates, variances, and reproductive values for all repeater populations compared with non-repeaters

\begin{tabular}{|c|c|c|c|}
\hline & $m-1$ & $\sigma^{2}$ & Average $R_{0}$ \\
\hline & \multicolumn{2}{|c|}{$\beta=0.10$} & \multirow{3}{*}{0.7} \\
\hline Ct female non-repeaters & 0.4 & 0.45 & \\
\hline $\mathrm{Ct}$ male non-repeaters & 0.31 & 0.73 & \\
\hline Ct female repeaters & 0.84 & 1.77 & \multirow{2}{*}{1.09} \\
\hline $\mathrm{Ct}$ male repeaters & 0.64 & $\begin{array}{l}1.94 \\
.43\end{array}$ & \\
\hline Gc female non-repeaters & 0.37 & 0.47 & \multirow{2}{*}{0.77} \\
\hline Gc male non-repeaters & 0.43 & 0.66 & \\
\hline Gc female repeaters & 0.34 & 0.68 & \multirow{2}{*}{1.01} \\
\hline Gc male repeaters & 0.97 & $\begin{array}{l}1.36 \\
.50\end{array}$ & \\
\hline Co-infected female non-repeaters & 0.41 & 0.92 & \multirow{2}{*}{0.97} \\
\hline Co-infected male non-repeaters & 0.4 & 0.33 & \\
\hline Co-infected female repeaters & 0.93 & 2.61 & \multirow{2}{*}{1.41} \\
\hline Co-infected male repeaters & 0.53 & 0.71 & \\
\hline
\end{tabular}

The average duration time of chlamydial infection was 10 months, and for gonorrhea and co-infection, 55 days. $\mathrm{Ct}$, chlamydia; Gc, gonorrhoea. 
Table 2 Proportions of chlamydia, gonorrhea, and co-infected repeaters and non-repeaters in Manitoba, 1990-1992, by ethnic group, lowest household income quintile, and use of aliases

\begin{tabular}{llll}
\hline Group & $\begin{array}{l}\text { First Nations } \\
(\%)\end{array}$ & $\begin{array}{l}\text { Income } \\
<\$ C N D 29 \\
500(\%)\end{array}$ & $\begin{array}{l}\text { Used } \\
\text { alias (\%) }\end{array}$ \\
\hline Ct non-repeaters & 16.7 & 35.6 & 13.9 \\
Ct repeaters & 36 & 51.1 & 23.8 \\
Gc non-repeaters & 27.6 & 58.5 & 13.4 \\
Gc repeaters & 46.9 & 69.2 & 22.7 \\
Co-infected non-repeaters & 35.1 & 60.7 & 20.1 \\
Co-infected repeaters & 51.6 & 65 & 33.4 \\
\hline
\end{tabular}

Ct, chlamydia; First Nations, Canadian aboriginals; Gc, gonorrhoea.

were lower for each STI (table 1). $\mathrm{R}_{0}$ values for chlamydia non-repeaters were 0.7 , for gonorrhoea non-repeaters 0.77 , and co-infected non-repeaters 0.97. Chlamydia repeaters had an $\mathrm{R}_{0}$ value of 1.09 , gonorrhoea repeaters slightly lower at 1.01, and co-infected repeaters, who may be at the centre of the core, had a value of 1.41. Proportions of First Nations (North American Indians) increased along with the $\mathrm{R}_{0}$ values in both repeaters and non-repeaters, as did the use of aliases and the proportion of people living in areas with the lowest income quintile in Manitoba (table 2). These gradients suggest that a core group is a matter of degree, with core populations having various abilities to maintain different endemic infections.

The feasibility of constructing sexual networks (contact tracing trees) for all repeaters was also assessed. Because the network sizes had never before been explored using contact tracing data at the time of this research, estimations of sample sizes to show differences in network size were made using a small convenience sample, selected alphabetically, of the first 48 repeaters and 41 non-repeaters. The feasibility study yielded dyads and triads and one small network. In addition, a network of more than 900 individuals was discovered. The construction of the networks could not be computed, so work was halted after the network reached 900 individuals.

Figure 1 shows 126 people from the large network, by sex and type of infection. They surround "7957", who was the contact in the database named most often but who never became a case. He was named 13 times on different occasions by eight women, starting in January 1990 and ending in December 1992. By the network measure of degree centrality, this is the contact who was most important in this network. Women who named him were confirmed as having gonorrhoea or chlamydia, and three had both infections. While co-infected individuals comprised only $6 \%$ of all STI cases, $35 \%$ of cases in this region of the network had confirmed co-infection within the study period.

Figure $1 \mathrm{~B}$ is identical to fig $1 \mathrm{~A}$ but shows the proportion of repeaters present in the same portion of the large network. There is a higher concentration of repeaters $(51.8 \%)$ among the cases in this diagram than found in the total number of people with STIs reported to Manitoba Health during the same three year study period (30.5\%).

The research confirmed that the repeater populations had reproductive numbers which met the criteria for maintaining an epidemic. Constructing the networks of repeaters and their contacts illustrates the dyadic relation between the sexes, as it relates to the reproductive numbers shown in table 1 . The fact that $\mathrm{R}_{0}$ for co-infected men is less than for women may be because of interactions between infected men and the women, such as the one at the far right of fig 1B. In that case, "9955" did not require large numbers of partners to be an effective transmitter, as the effect of one partnership with "124798" amplified transmission to more than seven other men. In addition, the concentration of co-infected individuals with the highest reproductive numbers in this portion of the network suggests that this part of the network may form a core group.

\section{SEXUAL NETWORK ANALYSIS OF CHLAMYDIA AND} GONORRHOEA TRANSMISSION IN MANITOBA

Having established that sexual networks could be constructed from the contact tracing data, a study to describe the social, sexual, and microbiological aspects of disease transmission was proposed. The subjects were individuals infected with chlamydia with or without concurrent gonorrhoea during the six months from November 1997 until May $1998 .{ }^{18}$ In order to capture partnership information on contacts reported during the study period, we also included cases from August to October of 1997. This yielded a total 4544 contacts and case records which were analysed in Pajek (ㄷ 1996, Batagelj V, Mrvar A), revealing 1503 networks, ranging in size from two (dyads) to 82 people.

Most important was the discovery of geographical bridges in the networks, which were essential in connecting network members across vast distances. Only five of the larger networks $(n>9)$ had members who all lived within the same community, town, or city. Most large networks had at least one member living in Winnipeg, and the remainder contained people living in different communities in northern Manitoba. Travel to some of these communities is often restricted to air or boat only, and was common between communities $500 \mathrm{~km}$ or more away. A significant proportion of the sexual partnerships between people from different communities was also reported by Calzavara et al, whose research was completed in First Nations communities in Ontario. ${ }^{32}$

Analysis of sexual networks within Winnipeg refined ecological research by Blanchard et al, who classified forward sortation areas in the city of Winnipeg (where more than half the population of Manitoba reside) into core, adjacent, and periphery by levels of incidence of chlamydia. ${ }^{33}$ The diagram of the largest component in this network illustrates the transmission dynamics, where a single relationship between a "core" and "non-core" individual linked the remaining 18 people in the network, the majority of whom reside in peripheral areas of Winnipeg (fig 2 ). This relationship not only provides insight into bridge events, but also emphasises the fact that targeting high incidence populations on the basis of geography may not necessarily decrease infections, especially where conduits of infection into and out of the targeted populations remain unidentified and untreated. While the geographical approach to STI control is useful, it should be supplemented with programmes that include communities in which sex partners are commonly recruited. Data on where sex partners are recruited may be gathered using traditional survey techniques, or sociocentric or egocentric network research in populations with STI. It should be recognised that partners at high risk for STI may be recruited from different communities than partners at low risk for STI.

In addition to the above analyses, the chlamydia ompl gene from network members was sequenced to supplement our knowledge of routes of infection. A preliminary assessment of the agreement between the sexual partnerships and the genotypes was completed by drawing a sample of nine couples from whom specimens were obtained 12 days apart or less. Should couples who named each other have discordant genotypes, it would be necessary to question whether the contact tracing data could identify possible transmission routes. Eight of nine couples had matching genotypes, and one individual of the ninth couple had type E, while the other had an infection of type E mixed with another type. This was not surprising, as both partners reported three recent sex partners each. Thus where a partnership was reported, types were mostly concordant, but concordant types may not necessarily indicate that a sexual partnership existed between two or more people. 
A

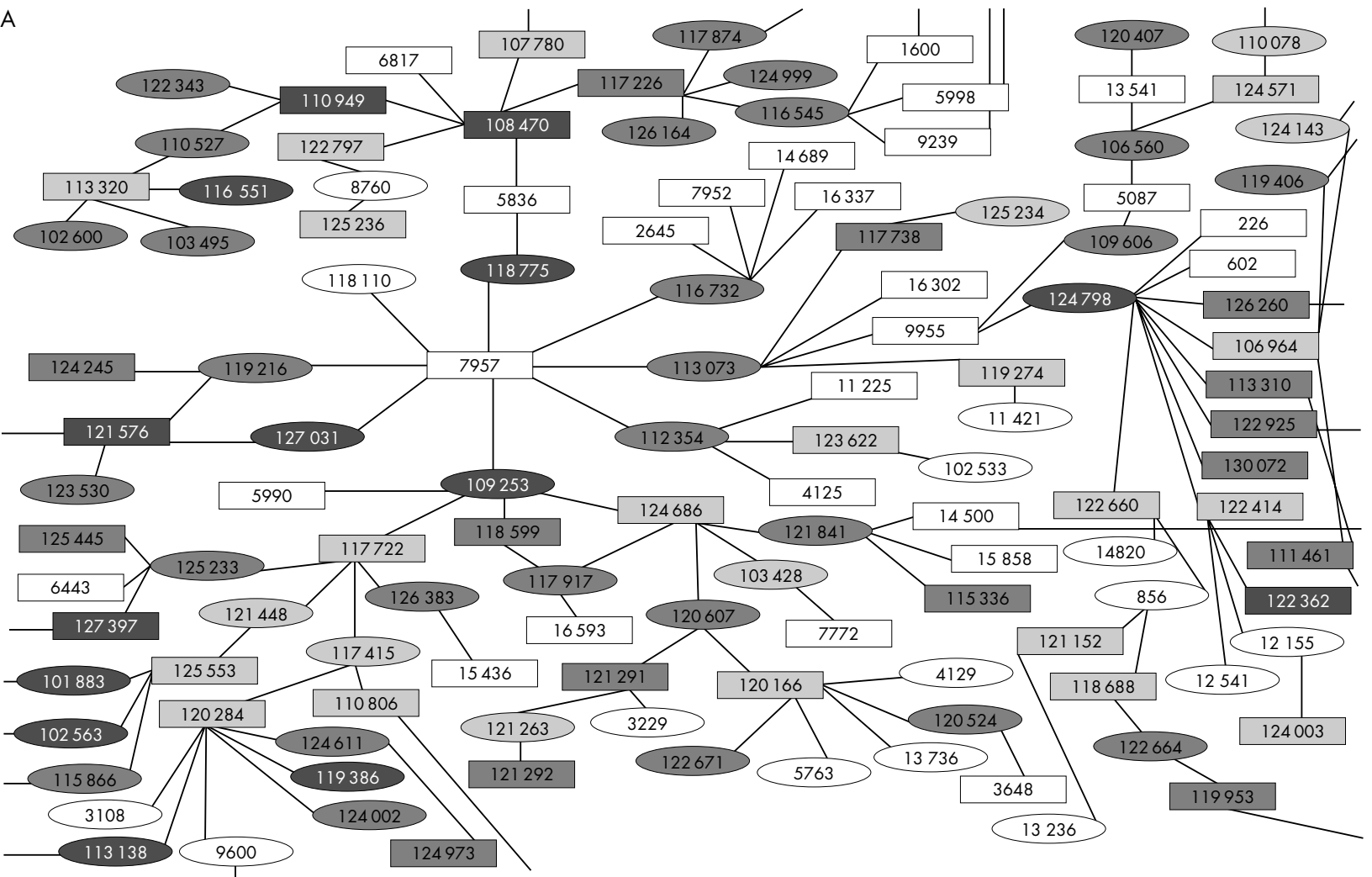

B

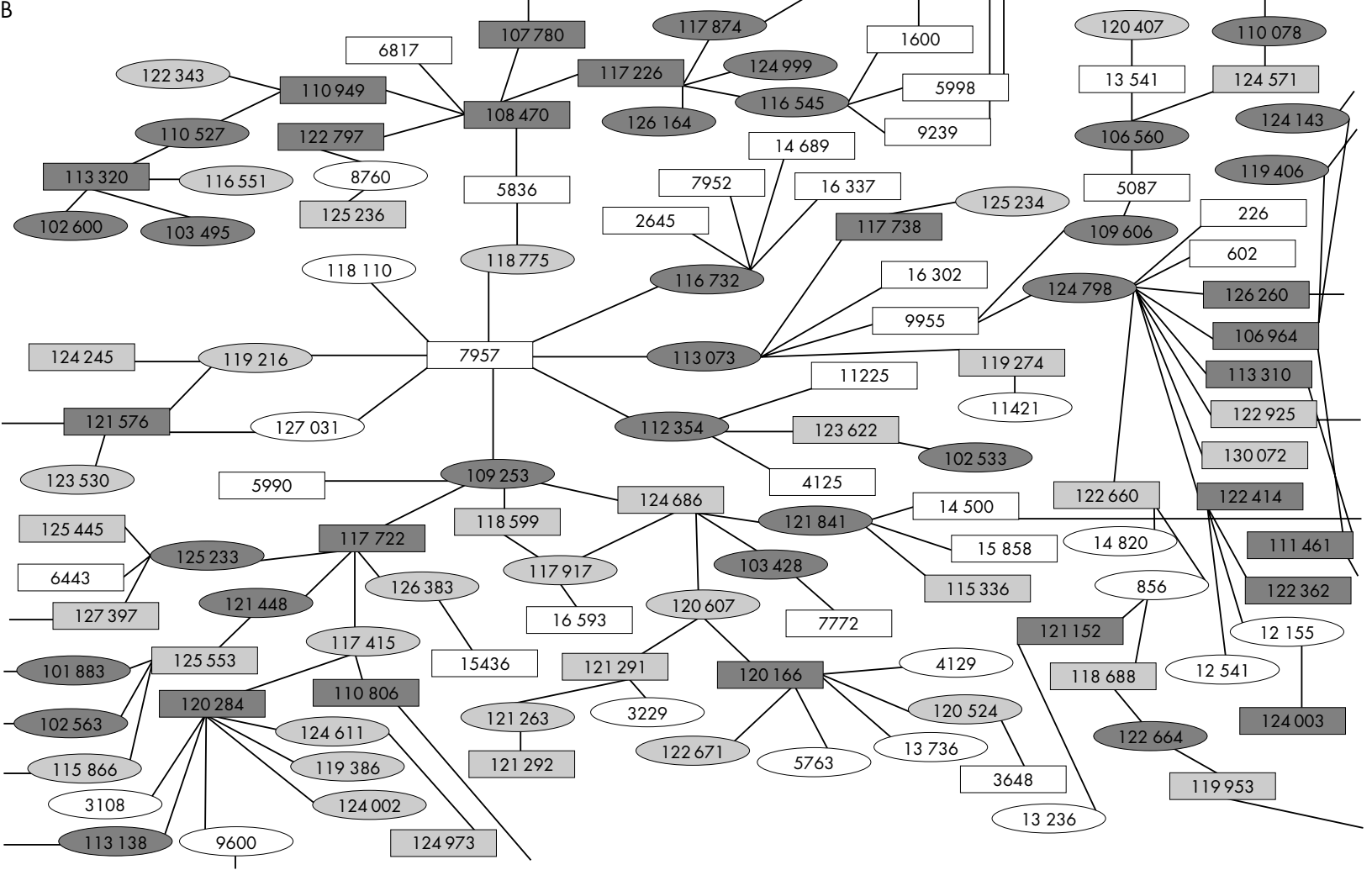

Figure 1 (A) Section of the large network showing 126 individuals, the upper limits of which were never established. Contacts who never had laboratory confirmed infection are shown in clear white; gonorrhea cases in pale grey; chlamydia in mid-grey; and co-infected individuals in dark grey. Male subjects are depicted by rectangles; female subjects by ellipses, and each node contains the individual's study identifier. (B) Section of large network of people $(n=126)$ with chlamydia and/or gonorrhea, the upper limits of which were never established, Manitoba 1990-92. Contacts who were never confirmed cases are shown in clear white; cases testing positive once are shown in orange, and those testing positive twice or more are shown in red. Male subjects are depicted by rectangles; female subjects by ellipses, each of which contain study identification numbers. 


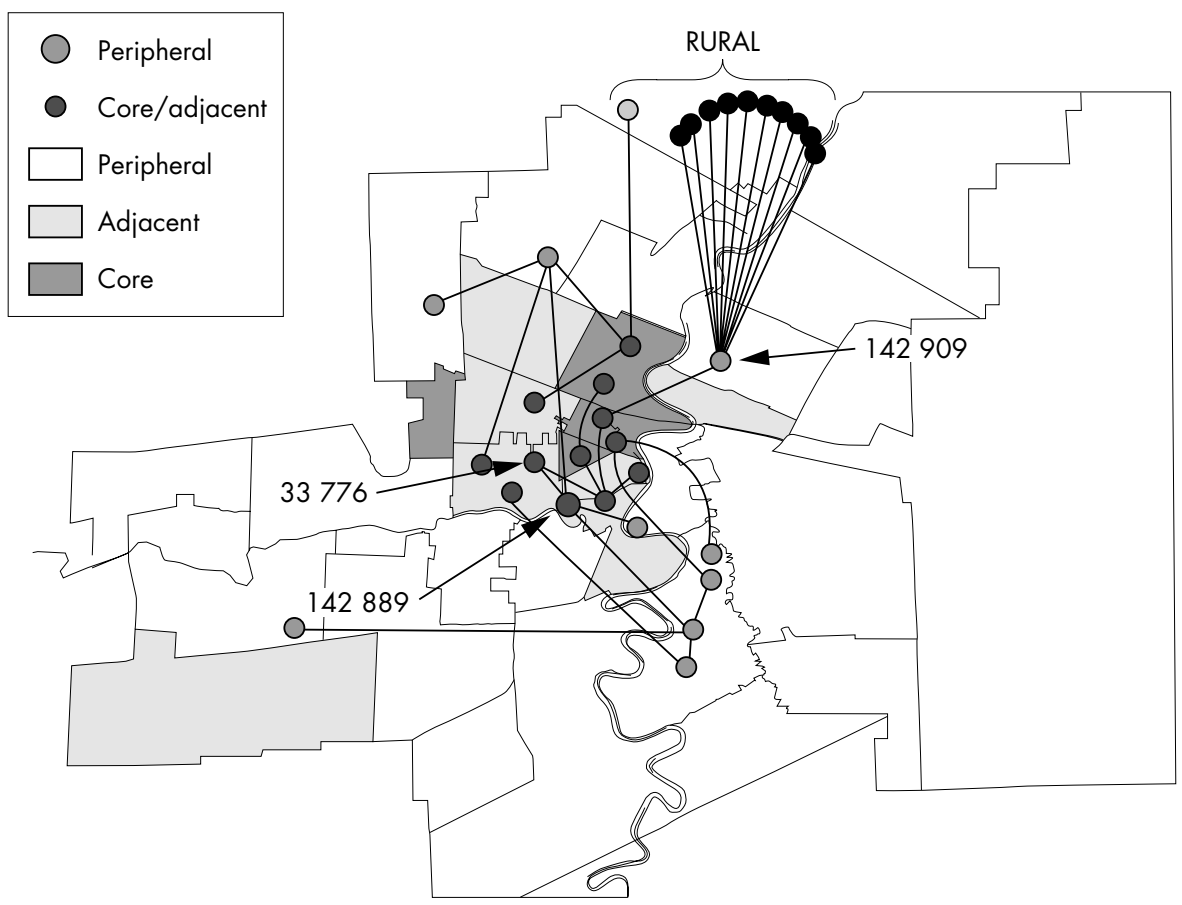

Figure 2 Sexual network patterns in Winnipeg. The diagram shows component 23, superimposed on a map of Winnipeg divided in to 34 forward sortation areas. Indicated by arrows, case 142909 connects the other members in the North with those in Winnipeg. The male contact 33 776, resided in the "core" area of Winnipeg, while his female partner 142889 resided in an adjacent area. She performs an important function as a bridge, as all of her partners but one, together with their partners, form a subnetwork in peripheral and adjacent areas of Winnipeg, rather than the core.

Chlamydia sequencing information will be of most value in interpreting transmission within larger networks, which may contain core groups.

A secondary objective in the above project on chlamydia sexual networks in Manitoba was to define the characteristics of clients who are likely to belong to large networks in order to facilitate future prospective studies. ${ }^{34}$ We evaluated three methods of sampling chlamydia positive individuals to determine which would yield the largest sexual networks. The groups of people sampled were as follows:

(1) a random sample of individuals $(n=120)$ with one positive test for chlamydia during the study period November 1997 to May 1998;

(2) individuals with more than one positive test during the same period $(\mathrm{n}=93)$;

(3) individuals who tested positive for both chlamydia and gonorrhoea during the study period $(\mathrm{n}=76)$;

(4) the last two groups of repeaters and co-infected people combined.

The number and sizes of the networks were compared against those constructed using information from the total dataset.
All four groups contained individuals who were members of significantly larger networks than those in the total population (table 3). Network size increased from the random sample to the repeaters, and was largest for the co-infected group. In addition, the number of networks decreased from $6.7 \%$ of the total networks represented in the random sample to $4.5 \%$ for the repeaters and 3.3\% for the co-infected group. Thus, constructing networks of the co-infected group revealed the highest proportion of large networks for the lowest number of individuals traced, and the highest proportion of large networks overall was accessed by sampling the repeater and co-infected groups.

Various factors may have affected average network sizes. Although the random sample of individuals ensured each individual an equal chance at being selected, it resulted in preferential selection of larger networks, purely because of the number of individuals within them. In the case of the repeaters, multiple opportunities to collect more complete data on individuals and their partners may have facilitated partner notification. In addition, increased recognition of symptoms on repeat infections could have resulted in the client presenting for care. Public health practice patterns may have increased the priority, thoroughness, and effort taken to locate

Table 3 Comparison of the network sizes yielded by all four samples to those of the total population, showing the mean, median, proportion of total networks, and proportion of large networks of 10 or more people $(n=23)$

\begin{tabular}{llllll}
\hline Group & Mean & Median & $\begin{array}{l}\text { Per cent of } \\
\text { total }\end{array}$ & $\begin{array}{l}\text { Per cent large networks } \\
\text { (>9 people) }\end{array}$ & p Value \\
\hline Total population & 2.7 & 2 & 100 & 100 & \\
Random sample & 3.7 & 2 & 6.7 & 26.1 & $<0.001$ \\
Repeaters & 5.5 & 3 & 4.5 & 30.4 & $<0.001$ \\
Co-infected & 7.3 & 4 & 3.3 & 34.8 & $<0.001$ \\
Co-infected and repeaters & 5.6 & 3 & 7.2 & 47.8 & $<0.001$ \\
\hline
\end{tabular}


individuals infected with two notifiable diseases, compared with only one. Also, the greater likelihood of co-infected individuals experiencing symptoms and therefore presenting for care may also have influenced network size. However, by far the greatest source of bias is that of underreported sex partners, ${ }^{35}$ which underestimates the number of partners to whom infection was potentially transmitted, and therefore also underestimates network size. Thus the numbers presented in tables 1 and 3 represent conservative estimates of numbers of partners, reproductive numbers, and network sizes.

Despite these limitations, the fact that co-infected individuals had the highest proportion of large networks for the lowest number traced is consistent with the calculation of $\mathrm{R}_{0}$ values in the first study reported, where the reproductive number for the co-infected group was higher than any other, suggesting that they are most capable of maintaining the endemicity of an STI within a population. This ratio of the few cases who are directly and indirectly sexually connected with other members of a large network reflects early work done in Colorado, ${ }^{36}$ where gonorrhoea "transmitters" with two or more sexual contacts ( $22 \%$ of the total population of cases) were found to be members of large "lots" (sexual networks), producing seven times the average number of person-days of infection. In addition, the vast majority of people in the interior of the large networks in the chlamydia network study had more than one sex partner to whom infection could have been transmitted, and at least one sex partner with documented chlamydial infection during the study period. While laboratory-confirmed infection in both the index case and at least one other named partner indicates membership in a core group, proof that the organism is transmitted to the partner by a specific index case is problematic because previous infection of a sex partner by another index case may already have taken place. Yorke et al first defined the core in $1978^{12}$ as being a group in which a "significant" number of pre-emptive infections takes place when infectious individuals contact people who are already infected by different sources. These investigators also state that pre-emptive infections are significant when the prevalence of infection in the group exceeds $20 \%$. All of our networks of 10 people or more exceed this prevalence rate, most by a substantial margin. ${ }^{18}$

\section{NETWORK CHARACTERISTICS IN LOW INCIDENCE ENDEMIC AREAS}

Future research and prevention strategies should take into account the combined effects of the organism's biological evolution and the pressure of the intervention programme on the organism to "find" new ecological niches in which it can flourish. In the case of sexually transmitted pathogens, sexual networks constitute ecological niches. As outlined by Wasserheit and Aral, there is often an initial downward trend as the prevention programme takes effect, followed by a less steep decrease as infections and reinfections in the core are detected, and finally a horizontal line with oscillations around the mean. These oscillations indicate organisms breaking through to various new ecological niches or subnetworks in which they can reproduce, although perhaps on a smaller scale. ${ }^{36} 37$

It is likely that in the last phase of low incidence endemicity, investigation will reveal both small and large networks such as those we found. It is also likely that the large networks would contain members who had many sex partners, or who would form particular structures within the network; these people may be members of a core group. Obviously, the majority of people in either a small or a large network with one or two partners, where a path terminates, are probably incapable of transmitting disease effectively, and cannot be considered to be core group members. The construction of networks and the calculations of $\mathrm{R}_{0}$ indicate that sexual networks are similar in structure to the layers of an onion, the centre of which contains the most effective transmitters (in this case of gonorrhoea and chlamydia simultaneously) while adjacent members transmit only chlamydia or gonorrhoea, and those on the outer edges of the network probably do not transmit infection at all. Owing to underreporting, underdiagnosis, or anonymous sex partners, the small networks are probably the peripheral parts of a larger network containing the core. ${ }^{38}$ While the staging of historical phases of an epidemic is essential in defining strategies for prevention, the designation of different "spread," "maintenance," and "die out" networks oversimplifies the results of network analysis presented here. We believe that all these functions may be present in one sexual network, and that many such sexual networks exist within the Manitoba population.

\section{STI RESEARCH AND PREVENTION: FUTURE DIRECTIONS}

Monitoring incidence trends closely in the later epidemic phases is essential in identifying outbreaks and oscillations. This will facilitate prompt, thorough investigations after an unusual rise in numbers has been detected. The traditional surveillance approach combined with network investigation may reveal networks that contain effective STI transmitters. Where only small networks are found, without obvious links to the core, active investigation of bridging events which seed infection from the core into the subnetworks is warranted. This may be the only way of accessing the core groups in later stages of the epidemic when transmission becomes rare. The network approach will also help in clarifying which individuals, named initially as contacts of the first recognised cases, are likely transmitters or source cases. When the network is plotted along a time line, people at high priority for follow up are contacts named by cases for the period just before the onset of symptoms, known as "upstream contacts", 31 as they are people who have most probably transmitted an STI to at least one other person, and who remain undiagnosed and untreated. They should be placed at a higher priority than those people to whom infection was transmitted, as they may not play a role in transmitting to others.

It is important to note that unusual rises in incidence may indicate new ecological niches which fall outside of our traditionally designated "target groups". The syphilis outbreak network described by Rothenberg et al, ${ }^{39}$ in which the demographic characteristics of the people were unlike those of the traditional targeted populations, indicates that this may have already occurred. Alternatively, prevention programmes may have been successful at eliminating an organism from a core group or network, but the network remains fertile ground for any reintroduction from an external source or bridge, such as in the reintroduction of syphilis in Vancouver, where patients have been largely poor, minority, inner city people connected with the sex and drug trades. ${ }^{4041}$

The extensive geographical spread of the networks across the whole province of Manitoba also has immediate practical implications for prevention programmes. Public health services have been fragmented into regions within many provinces in Canada, despite the fact that adequate partner notification, location, testing, and treatment require a centralised data system and a coordinated approach to contact follow up, regardless of the locations of cases and contacts.

In order to support effective interventions in low incidence endemic areas, research should focus on developing indicators of core group membership. Many network measures of structure and individual position exist, and more are being developed, all of which should be reviewed for their value in predicting transmission. ${ }^{39}{ }^{42}$ Predictors of core group membership derived from mathematical models alone may not be adequately sensitive or specific in defining core individuals, 
and as the epidemics become smaller and numbers decrease, statistical measures will become less reliable. Additionally, mathematical models may not take network structure into account when predicting persistence of a pathogen. ${ }^{43}$ Finally, the degree to which networks are core groups, where the average member transmits infection to more than one other person $\left(\mathrm{R}_{0}>1\right)$, warrants further investigation.

It is possible that the mathematical and network investigations into core group membership will not yield the same results, but the exercise itself will provide insights into transmission mechanisms and confirm hypotheses. The attraction of the network approach is its intuitiveness in public health settings, and relative ease of application, given increasing access to computed data. Despite its limitations, it presents empirical data from which hypotheses can be generated and solved, in addition to those already inspired by mathematical epidemiologists.

\section{Authors' affiliations}

A M Jolly, Division of Sexual Health Promotion and STD Prevention and Control, Centre for Infectious Disease Prevention and Control, Health Canada, Ottawa, Ontario, Canada

J L Wylie, Department of Medical Microbiology, University of Manitoba and Cadham Provincial Laboratory, Manitoba Health, Winnipeg, Manitoba, Canada

\section{REFERENCES}

1 Rothenberg RB, Potterat JJ. Strategies for management of sex partners. In: Holmes KK, et al, eds. Sexually transmitted diseases, 2nd ed. New York: McGraw-Hill, 1990: 1081-6.

2 Division of STD Prevention and Control. Sexually transmitted diseases in Canada: 1996 Surveillance Report. Can Commun Dis Rep 1998;25S1:1-31.

3 Division of STD/HIV Prevention. Sexually transmitted disease surveillance 1992. Atlanta, Georgia: Centers for Disease Control and Prevention, U.S. Department of Health and Human Services, Public Health Service, 1993:13-24.

4 Bureau of Communicable Disease Epidemiology. 1989 Canadian guidelines for screening for Chlamydia trachomatis infection. Can Dis Wkly Rep 1989;15:1-11.

5 Over M, Piot P. Human immunodeficiency virus infection and other sexually transmitted diseases in developing countries: public health importance and priorities for resource allocation. J Infect Dis 1996;174:S162-75

6 Wasserheit JN, Aral SO. The dynamic topology of sexually transmitted disease epidemics: implications for prevention strategies. J Infect Dis 1996; 174:S201-13

7 Herrmann B, Egger M. Genital Chlamydia trachomatis infections in Uppsala County, Sweden, 1985-1993: declining rates for how much longer? Sex Transm Dis 1995;22:253-60.

8 Sellors JW. Screening for chlamydial infection. Taking stock [editorial]. Can Fam Physician 1995;41:188-92,196-9.

9 Division of STD Prevention and Control. Sexually transmitted disease surveillance 1997. Atlanta, Georgia: Centers for Disease Control, 1998

10 CDC Unit, Public Health Branch. Notifiable diseases Manitoba; annual review, 1996. Winnipeg, Manitoba, Manitoba Health, 1996:1-53.

11 Thomas JC, Tucker M. The development and use of the concept of a sexually transmitted disease core. J Infect Dis 1996;174(suppl 2):S134-43.

12 Yorke JA, Hethcote HW, Nold A. Dynamics and control of the transmission of gonorrhea. Sex Transm Dis 1978;5:51-6.

13 Anderson RM, May RM. Infectious diseases of humans; dynamics and control, 1 st ed. Oxford: Oxford University Press, 1992.

14 Brunham RC, Plummer FA. A general model of sexually transmitted disease epidemiology and its implications for control. Med Clin North Am 1990;74:1339-52
15 Anderson RM, May RM. Infectious diseases of humans; dynamics and control, 1st ed. Oxford: Oxford University Press, 1992:234.

16 Wallace R. Social disintegration and the spread of AIDS. II. Meltdown of sociographic structure in urban minority neighborhoods. Soc Sci Med 1993;37:887-96.

17 Elliott LJ, Blanchard JF, Beaudoin CM, et al. Geographical variations in the epidemiology of bacterial sexually transmitted infections in Manitoba, Canada. Sex Tansm Infect 2002;78(suppl): i 139-44.

18 Wylie JL, Jolly AM. Sexual network analysis of chlamydia and gonorrhea transmission in Manitoba, Canada. Sex Transm Dis 2000;28:14-24.

19 Cleavenger RL, Juckett RG, Hobbs GR. Trends in chlamydia and other sexually transmitted diseases in a university health service. J Am Coll Health 1996;44:263-5.

20 Communicable Disease Control and Manitoba Health. Sexually transmitted disease control guidelines. Winnipeg: Manitoba Health, 1994:18-32.

21 Beller M, Middaugh J, Gellin B, et al. The contribution of reinfection to gonorrhea incidence in Alaska, 1983 to 1987. Sex Transm Dis 1992;19:41-6.

22 Blackwood LG. Repeated infections with Neisseria gonorrhoeae in a population of Alaska natives. Sex Transm Dis 1982:9:89-92.

23 Brooks GF, Darrow WW, Day JA. Repeated gonorrhea: an analysis of importance and risk factors. J Infect Dis 1978;137:161-9.

24 Oh MK, et al. Risk for gonococcal and chlamydial cervicitis in adolescent females: incidence and recurrence in a prospective cohort study. J Adolesc Health 1996;18:270-5.

25 Anderson RM. The transmission dynamics of sexually transmitted diseases: the behavioral component. In: Wasserheit JN, Aral SO, Holmes $\mathrm{KK}$, et al, eds. Research issues in human behavior and sexually transmitted diseases in the AIDS era. Washington DC: American Society for Microbiology, 1991:38-60

26 Stigum $\mathbf{H}$, Magnus $P$, Bakketeig LS. Effect of changing partnership formation rates on the spread of sexually transmitted diseases and human immunodeficiency virus. Am J Epidemiol 1997;145:644-52.

27 McCormack WM et al. Fifteen month follow-up study of women infected with Chlamydia trachomatis. N Engl J Med 1979;300:123-5.

28 Blount JH. A new approach for gonorrhea epidemiology. Am J Public Health 1972;62:710-12.

29 Henderson RH. Control of sexually transmitted diseases in the United States - a federal perspective. Br J Vener Dis 1977:53:211-15.

30 Potterat JJ, Phillips L, Rothenberg RB, et al. Gonococcal pelvic inflammatory disease: case-finding observations. Am J Obstet Gynecol 1980;138: 1101-4

31 Hethcote HW, Yorke JA, Nold A. Gonorrhea modeling: a comparison of control methods. Math Biosci 1982;58:93-109.

32 Calzavara LM, Bullock SL, Myers T, et al. Sexual partnering and risk of HIV/STD among aboriginals. Can J Public Health 1999;90:186-91.

33 Blanchard JF, et al. The evolving epidemiology of chlamydial and gonococcal infections in response to control programs in Winnipeg Canada. Am J Public Health 1998;88: 1496-502.

34 Jolly AM, Wylie JL. Sampling individuals with large sexual networks: an evaluation of four approaches. Sex Transm Dis 2001;28:200-7.

35 Brewer DD, Garrett SB, Kulasingam S. Forgetting as a cause of incomplete reporting of sexual and drug injection partners. Sex Transm Dis 1999;26:166-76.

36 Potterat JJ, et al. Gonorrhea as a social disease. Sex Transm Dis $1985 ; 12: 25-32$.

37 Klovdahl AS. Social networks and the spread of infectious diseases: the AIDS example. Soc Sci Med 1985;21:1203-16

38 Jolly AM, Muth SQ, Wylie JL, et al. Sexual networks and sexually transmitted infections: a tale of two cities. J Urban Health $2001 ; 78: 433-45$

39 Rothenberg RB, et al. Using social network and ethnographic tools to evaluate syphilis transmission. Sex Transm Dis 1998;25:154-60.

40 Rekart ML, et al. Mass treatment/prophylaxis during an outbreak of infectious syphilis in Vancouver, British Columbia. Can Commun Dis Rep 2000;26:101-5.

41 Patrick DM, Rekart ML, Jolly A, et al. Heterosexual outbreak of infectious syphilis: epidemiological and ethnographic analysis and implications for control. Sex Tansm Infect 2002;78(suppl):i 163-8.

42 Rothenberg RB, et al. Choosing a centrality measure: epidemiologic correlates in the Colorado Springs study of social networks. Social Networks 1995:17:273-97.

43 Brunham RC. Core group theory: a central concept in STD epidemiology. Venereology 1997;10:34-9 